\title{
Estimativa do filocrono em calêndula
}

\author{
Estimating the phyllochron in calendula
}

\author{
Jana Koefender ${ }^{\text {* }}$ Nereu Augusto Streck ${ }^{\mathrm{II}}$ Galileo Adeli Buriol ${ }^{\mathrm{II}}$ Roberto Trentin $^{\mathrm{IV}}$
}

\section{RESUMO}

O objetivo deste trabalho foi estimar o filocrono em plantas de calêndula (Calendula officinalis L.) cultivadas em diferentes épocas de semeaduras. Realizaram-se três épocas de semeaduras: 06/04/2005, 23/06/2005 e 03/10/2006, no interior de uma estufa plástica com área de $240 \mathrm{~m}^{2}$, instalada no campus da Universidade Federal de Santa Maria, UFSM. A semeadura foi realizada em vasos plásticos com capacidade volumétrica para cinco litros. O delineamento experimental foi inteiramente casualizado com 320 vasos em cada época, sendo distribuído em quatro fileiras de 80 vasos cada. Em uma planta de oito vasos de cada quatro fileiras, fez-se a contagem, semanalmente, do número de folhas emergidas da haste principal e na primeira haste lateral. Os dados do número de folhas foram correlacionados com a soma térmica diária acumulada, considerando-se uma temperatura base de $8^{\circ} \mathrm{C}$. Constatou-se que o filocrono variou com a época de semeadura, sendo o menor valor igual $15,9^{\circ} \mathrm{C}$ dia folha ${ }^{-1}$, obtido na $2^{a}$ época de semeadura e o maior, igual a $24,5^{\circ} \mathrm{C}$ dia folha ${ }^{-1}$, na $1^{a}$ época na haste principal e $48,9^{\circ} \mathrm{C}$ dia folha ${ }^{-1}$ na semeadura de outubro na primeira haste lateral.

Palavras-chave: soma térmica, Calendula officinalis $L$, aparecimento de folhas.

\section{ABSTRACT}

The objective of this study was to estimate the phyllochron in calendula (Calendula officinalis L.) grown in different sowing dates. Three sowing dates (06/04/2005, 23/06/ 2005 and 03/10/2006) were performed inside a $240 \mathrm{~m}^{2}$ plastic greenhouse at the Campus of the Federal University of Santa Maria, RS, Brazil. Sowing was in five liter plastic pots. The experimental design was a completely randomized with 320 pots on each sowing date and rows with 80 pots. The number of

\begin{abstract}
leaves in the main stem and oi the first lateral branch was counted on a weekly basis in one plant of eight pots. The number of leaves data was regressed against accumulated thermal time, assuming a base temperature of $8^{\circ} \mathrm{C}$. The main stem phyllochron varied with sowing date, with the lowest value $\left(15.9^{\circ} \mathrm{C}\right.$ day leaf $\left.{ }^{1}\right)$ obtained on the second sowing date, and the greatest value $\left(24.5^{\circ} \mathrm{C}\right.$ day leaf $\left.{ }^{-1}\right)$ obtained on the first sowing date. The greatest phyllochron on the first order lateral branch was $48.9^{\circ} \mathrm{C}$ day leaf ${ }^{-1}$ in the October sowing date.
\end{abstract}

Key words: thermal time, Calendula officinalis L, leaf appearance.

\section{INTRODUÇÃO}

O cultivo da calêndula (Calendula officinalis L.), planta medicinal, encontra-se em expansão no Brasil. Suas folhas e caules são, às vezes, consumidos como salada, mas são as flores, frescas ou secas, as partes da planta mais utilizadas em virtude, principalmente, das suas propriedades medicinais (DELLA LOGGIA et al., 1994; KALVATCHCHEV et al.,1997) e cosméticas (BAUMANN, 2003).

Além da importância medicinal, estudos comprovam que as sementes de calêndula contêm $20 \%$ de óleo e deste $59-60 \%$ são de ácido calêndico (CROMACK \& SMITH, 1998), que possui alta viscosidade (MUUSE et al., 1992), similar ao tung (Aleuritis fordii) (DERKSEN et al., 1995), utilizado na fabricação de tintas e cosméticos.

'Programa de Pós-graduação em Agronomia, Universidade Federal de Santa Maria (UFSM). Universidade de Cruz Alta (UNICRUZ),

Centro de Ciências Agrárias. Rua Andrade Neves, 308, 98025-810, Centro, Cruz Alta, RS, Brasil. E-mail: jkoefender@yahoo.com.br.

*Autor para correspondência.

"Departamento de Fitotecnia, Centro de Ciências Rurais (CCR), UFSM, Santa Maria, RS, Brasil.

IIICentro Universitário Franciscano (UNIFRA) e Departamento de Fitotecnia, CCR, UFSM, Santa Maria, RS, Brasil.

${ }^{\text {Iv }}$ Curso de Agronomia, CCR, UFSM, Santa Maria, RS,Brasil. 
O potencial fitoterápico da calêndula está comprovado cientificamente. Entretanto, questões básicas de manejo da cultura, como épocas de semeadura, que proporcionam distintas disponibilidades de radiação solar, temperatura do ar e precipitação pluviométrica ao longo do ciclo de desenvolvimento das plantas e que, por sua vez, afetam de forma distinta seu crescimento, desenvolvimento e sua taxa de aparecimento de folhas, carecem de estudos. Dessa forma, é importante que, juntamente aos estudos de produtividade, de compostos com propriedades medicinal e cosmética e de conteúdo de óleo essencial, sejam determinadas suas exigências bioclimáticas.

$\mathrm{O}$ registro do aparecimento de folhas em uma das hastes da planta é uma excelente medida de tempo fisiológico, o qual está associado ao momento de ocorrência dos diferentes estágios de desenvolvimento e ao início da ramificação lateral em diversas espécies vegetais, bem como à evolução da área foliar da planta. Esta tem relação direta com a interceptação de radiação solar, produção de fotoassimilados e produtividade biológica (STRECK et al., 2003b). Uma maneira de calcular o aparecimento de folhas na haste principal de plantas é por meio da determinação do filocrono, definido como o intervalo de tempo necessário para a emissão de folhas consecutivas (KLEPPER et al., 1982; FRANK \& BAUER, 1995; HERMES et al., 2001).

O método da soma térmica tem sido usado, freqüentemente, para representar o efeito da temperatura do ar sobre o desenvolvimento das plantas (JEFFERIES et al., 1987). Esse método simples é uma medida mais eficaz de tempo biológico que dias do calendário civil ou dias após a semeadura (STRECK, 2002). Utilizando-se a soma térmica como medida de tempo em plantas, o filocrono corresponde aos grausdia necessários para o aparecimento de uma folha $\left({ }^{\circ} \mathrm{C}\right.$ dia folha-1).

Não foram encontrados, na bibliografia, resultados de medida ou estimativa da velocidade de emissão de folhas em calêndula. Em vista disso, objetivou-se, neste estudo, estimar o filocrono em plantas de calêndula cultivadas em diferentes épocas de semeaduras, em Santa Maria, RS.

\section{MATERIAL E MÉTODOS}

Realizaram-se três épocas de semeadura da calêndula (06/04/2005, 23/06/2005 e 03/10/2005) no interior de uma estufa plástica instalada no Campo Experimental do Departamento de Fitotecnia da Universidade Federal de Santa Maria, Santa Maria, RS (latitude: $29^{\circ} 43$ 'S, longitude: $53^{\circ} 43^{\prime} \mathrm{W}$ e altitude: $95 \mathrm{~m}$ ). Utilizou-se a cultivar "Bonina Sortida", com flores de variadas cores.
A estufa plástica possuía dimensões de $10 \mathrm{~m} \times 24 \mathrm{~m}$, altura de $3 \mathrm{~m}$ na cumieira e $2 \mathrm{~m}$ de pé direito e a cobertura, as laterais e as frontais foram fabricadas com filme de polietileno de baixa densidade (PEBD), transparente e de $100 \mu \mathrm{m}$ de espessura. A ventilação interna foi realizada por meio da abertura e do fechamento das cortinas laterais, confome as condições meteorológicas do dia. Em dias quentes, as cortinas eram abertas e fechadas, respectivamente, em torno das $8 \mathrm{~h}$ e $18 \mathrm{~h}$ Em dias frios, nublados e/ou com nevoeiros, elas eram abertas em torno das $10 \mathrm{~h} \mathrm{e}$ fechadas, aproximadamente, às $15 \mathrm{~h} \mathrm{e}$, em dias com precipitação pluviométrica, elas permaneciam fechadas todo o dia.

O delineamento experimental foi inteiramente casualizado, com 32 plantas. A semeadura foi realizada em vasos de plástico de cor preta, com $0,25 \mathrm{~cm}$ de diâmetro, capacidade volumétrica para cinco litros e preenchidos com substrato comercial Plantmax. Foram semeadas sete sementes em cada vaso. Quando cerca de $50 \%$ das plântulas tinham mais de seis folhas, fez-se o raleio deixando-se quatro delas por vaso. Os vasos foram distribuídos no sentido do comprimento da estufa, lado a lado, em quatro fileiras de 80 vasos cada, totalizando 320 vasos. Logo após a emergência, foram marcados oito vasos a cada quatro fileiras e uma planta em cada um destes vasos, totalizando 32 plantas. Nessas plantas, foi contado o número de folhas na haste principal e na primeira haste lateral emergida, uma a duas vezes por semana, até o final do período de emissão de folhas. Foi considerada como folha visível quando a folha apical tinha pelo menos $2 \mathrm{~cm}$ de comprimento.

A umidade do substrato nos vasos foi mantida, permanentemente, próximaà capacidade de campo por meio de irrigação, utilizando uma mangueira plástica de $1,5 \mathrm{~cm}$ de diâmetro, com um dispositivo para gotejamento. As plantas foram fertirrigadas com uma solução nutritiva com a seguinte composição de sais macronutrientes, em mmol L${ }^{1}$, recomendada para o cultivo do tomateiro (ANDRIOLO, 1999): 6,7 de $\mathrm{KNO}_{3}, 1,3$ de $\mathrm{KH}_{2} \mathrm{PO}_{4}, 6,0 \mathrm{de} \mathrm{Ca}\left(\mathrm{NO}_{3}\right)_{2}$ e $1,8 \mathrm{de}$ $\mathrm{MgSO}_{4}$. Os micronutrientes foram adicionados conforme solução proposta por Jeannequin (1987), citado porANDRIOLO(1999).

A temperatura do ar foi registrada com o auxílio de um termohigrógrafo, instalado no interior de um miniabrigo de madeira pintado de branco, a 1,5m acima dos vasos. Os valores de temperatura foram obtidos dos termohigrogramas a cada duas horas. Com estes dados, calculou-se a temperatura média diária (Tm) somando-se os valores de temperatura coletados de duas em duas horas $(\mathrm{T} 0 \mathrm{~h}+\mathrm{T} 2 \mathrm{~h}+\ldots+\mathrm{T} 20 \mathrm{~h}+\mathrm{T} 22 \mathrm{~h})$ e dividindo-se pelo número de observações.

A soma térmica diária ( $\mathrm{STd}$ ) e acumulada (STa) desde a emergência de $50 \%$ das plantas do 
experimento foram calculadas, respectivamente, pelas equações:

$\mathrm{STd}=\mathrm{Tm}-\mathrm{Tb}$ e STa $=\sum \mathrm{STd}$ em que Tb é a temperatura base para o aparecimento de folhas.

Não foram encontradas referências na literatura sobre a temperatura base da calêndula. Assim, tendo em conta sua origem mediterrânea, (clima ameno), optou-se por uma temperatura base de $8^{\circ} \mathrm{C}$, pois, conceitualmente, a temperatura base é aquela abaixo da qual a planta não se desenvolve e se o fizer é a uma taxa muito reduzida (AZKUE \& PUCHE, 2003). Considerou-se como primeiro dia de contabilização da soma térmica quando houve $50 \%$ de emergência das plantas do experimento.

O filocrono foi estimado como o inverso do coeficiente angular da regressão linear entre número de folhas acumuladas na haste principal e na primeira haste lateral e a soma térmica acumulada (KLEPPER et al., 1982). Os dados de número final de folhas na haste principal e primeira haste lateral foram submetidos à análise de variância e as médias comparadas pelo teste de Tukey ao nível de 5\% de probabilidade de erro.

\section{RESULTADOS E DISCUSSÃO}

As temperaturas médias diárias do ar registradas no interior da estufa plástica durante o experimento foram de $18^{\circ} \mathrm{C}, 16,9^{\circ} \mathrm{C}$ e $24,5^{\circ} \mathrm{C}$, respectivamente, para as semeaduras em 6 de abril (época1), 23 de junho (época 2) e 3 de outubro (época
3) (Figura 1). Os maiores valores absolutos de temperaturas máximas foram registrados na semeadura da época $3\left(40,5^{\circ} \mathrm{C}\right)$ e de temperaturas mínimas de $2^{\circ} \mathrm{C}$ nas épocas $1 \mathrm{e} 2$. As temperaturas foram menores na época 2, mas, no decorrer do experimento, ocorreu um aumento na temperatura média (época 3), devido ao aumento do comprimento do dia e da perpendicularidade dos raios solares incidentes.

A relação entre o número médio de folhas (NF) acumuladas na haste principal e a soma térmica acumulada (STa) foi linear, com um coeficiente de determinação superior a 0,98 para todas as épocas (Figura 2a). Isso indica que a temperatura do ar foi o principal fator ambiental que determinou a emissão de folhas na calêndula. Estes resultados concordam com aqueles obtidos por outros pesquisadores que mostraram que o número de folhas é linearmente relacionado com o acúmulo de unidades térmicas desde a emergência da plântula (BAKER et al., 1986; MUCHOW \& CARBERRY, 1990; SLAFER et al., 1994).

O filocrono da haste principal variou em função da época de semeadura, sendo que o menor valor $\left(15,9^{\circ} \mathrm{C}\right.$ dia folha $\left.{ }^{-1}\right)$ foi estimado na época $2(23 /$ $06 / 2005)$ e o maior $\left(24,5^{\circ} \mathrm{C}\right.$ dia folha $\left.{ }^{-1}\right)$ na época $1(06 /$ 04/2005) (Figura 2a).

O filocrono na primeira haste lateral na época 3 foi superior ao da época $2\left(49,75^{\circ} \mathrm{C}\right.$ dia folha 1 e $26,46^{\circ} \mathrm{C}$ dia folha ${ }^{-1}$, respectivamente), comportamento diferente

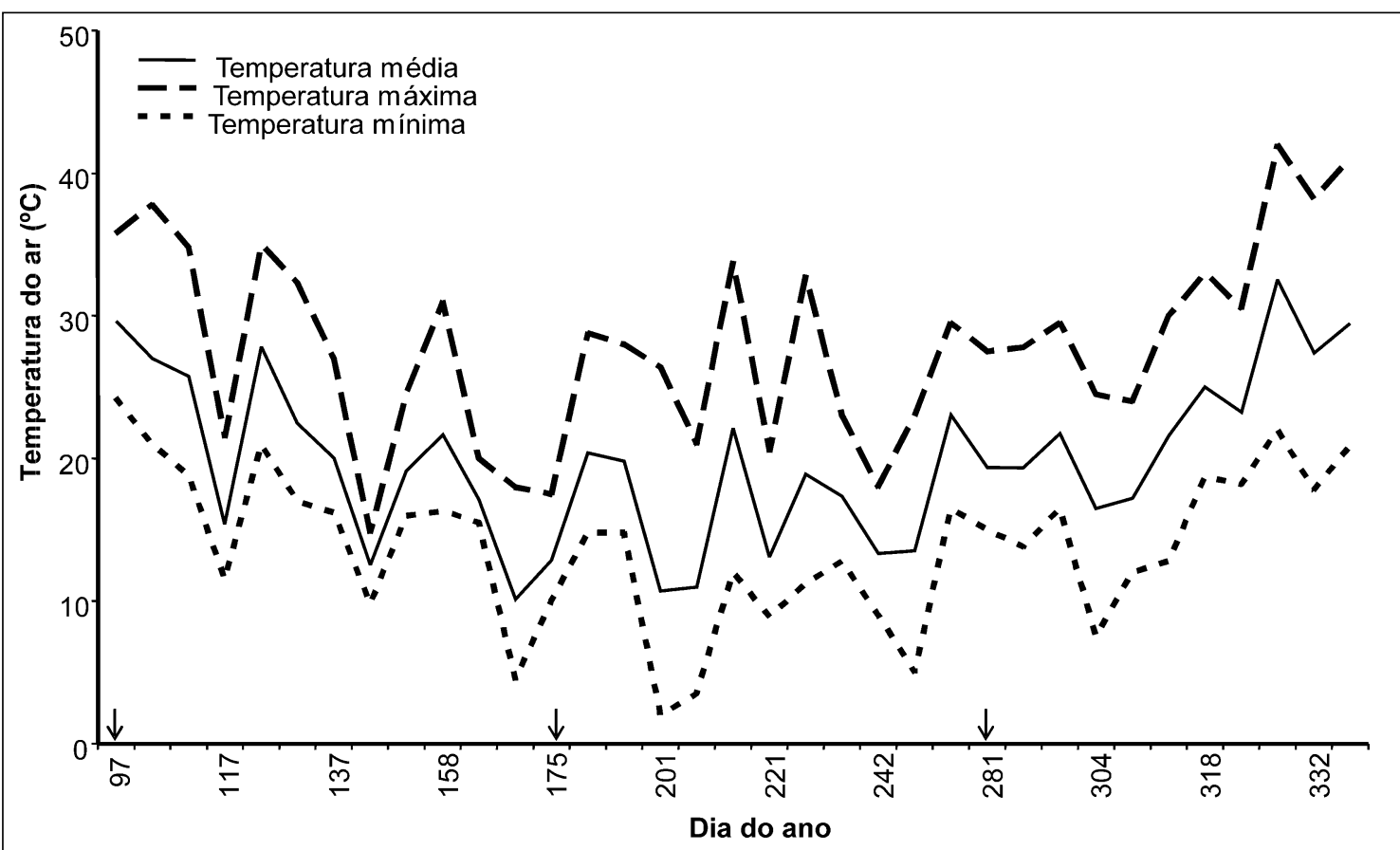

Figura 1 - Temperatura média, máxima e mínima diária do ar $\left({ }^{\circ} \mathrm{C}\right)$ registrada no interior da estufa plástica durante o período de 06/04/2005 a 28/11/2005. As setas indicam as datas de semeadura. Santa Maria, RS. 2005. 

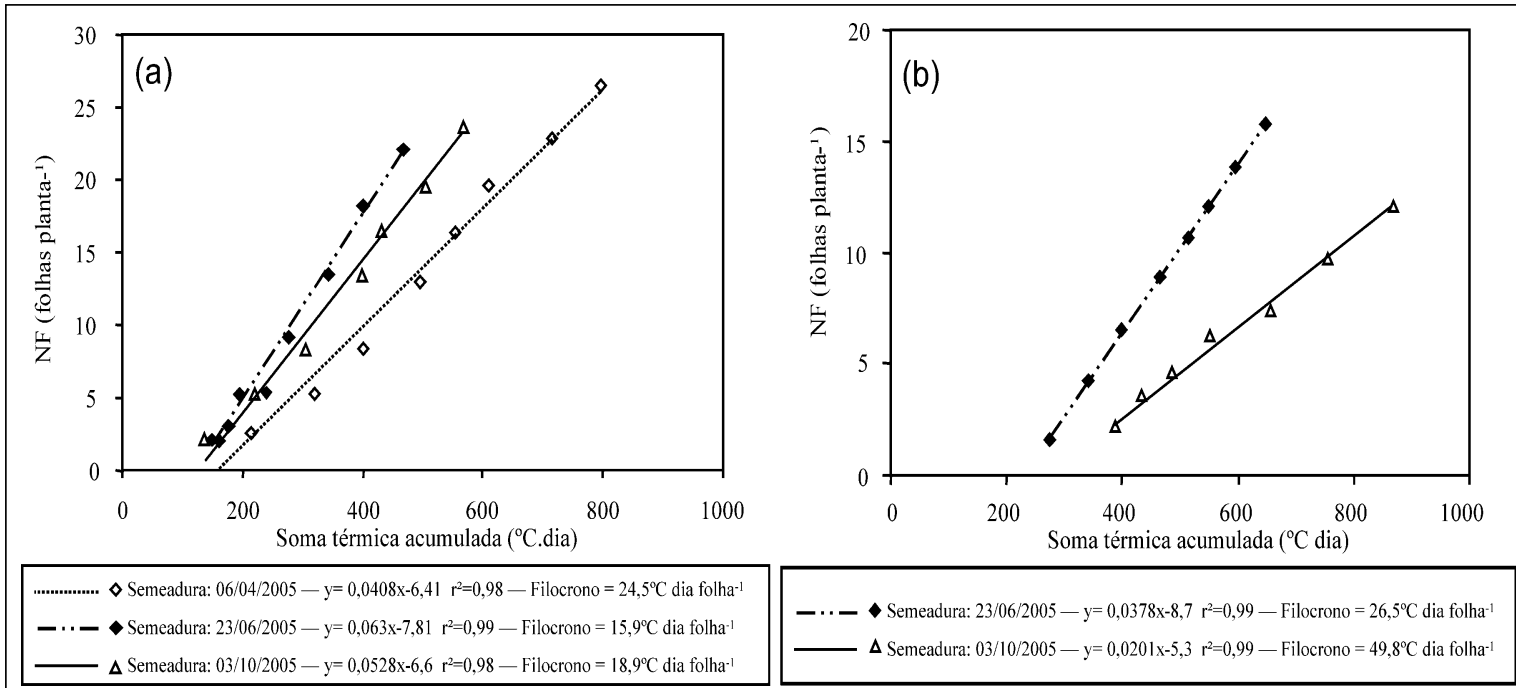

Figura 2 - Relação entre o número médio de folhas acumuladas na haste principal (a) e na haste lateral (b) e a soma térmica utilizada para a estimativa do filocrono em calêndula, cv. "Bonina Sortida", Santa Maria, RS, 2005.

quando comparado ao filocrono na haste principal para as mesmas épocas (Figura 2b). Isso evidencia que, para a emissão da primeira haste lateral, há necessidade térmica diferenciada. Diferenças de filocrono para um mesmo genótipo em diferentes épocas de plantio já foram relatados em batata (PAULA et al., 2005), em meloeiro (BAKER \& REDDY, 2001) e em distintas semeaduras de trigo, cevada e pastagens (FRANK \& BAUER, 1995; KIRBY, 1995). ELLIS \& RUSSEL(1984), KIRBY et al. (1985) e BAKER et al. (1986) verificaram que em trigo há efeito da época de semeadura na taxa de aparecimento foliar, indicando que, à medida que a semeadura é mais tardia, a soma de graus-dia requeridos para a emergência de uma folha é menor.

A variação do filocrono da calêndula em diferentes épocas de semeadura pode estar relacionada com fatores ambientais como o fotoperíodo e a temperatura do ar. No entanto, no presente estudo não se constatou influência do fotoperíodo no aparecimento de folhas na haste principal, pois o coeficiente de determinação da análise de regressão da relação entre o número de folhas acumuladas na haste principal e o fotoperíodo na emergência foi baixo $\left(r^{2}=0,29\right)$ e $\left(r^{2}=0,04\right)$ na fase vegetativa. BERTI et al. (2003), estudando o efeito de épocas de semeadura no rendimento de capítulos de diferentes genótipos de calêndula no Chile, verificaram um período mais curto entre a fase de emergência-floração na semeadura da época de 04/06/2003 que nas demais épocas, 05/08, 07/ 09 e 14/10. Segundo os autores, a explicação está no fato de a calêndula ser uma planta de dia longo e ter um baixo requerimento de horas luz para florescer, entre seis horas e meia e oito horas de luz.
Analisando-se as temperaturas médias durante a emissão de folhas na calêndula, observouse uma tendência de aumento do filocrono com a elevação da temperatura do ar $(\mathrm{y}=1,0022 \mathrm{x}+0,5581$; $\left.r^{2}=0,37\right)$. Semelhante tendência foi observada por PAULA et al. (2005) na cultura da batata cv "Asterix" cultivada em diferentes épocas, em que houve uma variação do filocrono de 22,2 a $35,5^{\circ} \mathrm{C}$ dia folha ${ }^{-1}$. Estes autores sugerem que essa resposta do filocrono à temperatura do ar seja resultado da relação linear entre temperatura do ar e emissão de folhas assumida no cálculo da soma térmica. O uso do método da soma térmica como medida de tempo tem limitações, pois a resposta mais realística do desenvolvimento vegetal à temperatura do ar é não-linear (STRECK et al., 2003ab; XUE et al., 2004), de modo que a relação linear da soma térmica pode levar a erros no cálculo. Assim, quando as semeaduras ocorrem em períodos em que as temperaturas não são consideradas ótimas para o desenvolvimento, o método da soma térmica poderá não ser apropriado.

$\mathrm{O}$ número final de folhas na haste lateral não foi influenciado pelas diferentes épocas de semeaduras (Tabela 1). Na análise estatística de contraste, verificou-se que a média do número final de folhas na haste principal da época $1(26,6)$ é significativamente maior em relação à média dos demais tratamentos $(22,8)$. Na haste principal das plantas de calêndula, nas semeaduras da época de junho e de outubro, o número total de folhas foi menor que na semeadura de abril.

\section{CONCLUSÃO}

A época de semeadura interfere no filocrono e no número de folhas na haste principal e na primeira haste lateral da calêndula.

Ciência Rural, v.38, n.5, ago, 2008. 
Tabela 1 - Número final de folhas na haste principal (NFFHP) e número final de folhas na primeira haste lateral (NFFHL) em plantas de calêndula (Calendula officinalis) cultivadas em diferentes épocas de semeadura. Santa Maria, RS, 2005.

\begin{tabular}{lll}
\hline Data de semeadura & NFFHP & NFFHL \\
\hline $06 / 04 / 2005$ & $26,63 \mathrm{a}^{*}$ & - \\
$23 / 06 / 2005$ & $23,06 \mathrm{~b}$ & $21,52 \mathrm{a}$ \\
$03 / 10 / 2005$ & $24,84 \mathrm{a} \mathrm{b}$ & $21,00 \mathrm{a}$ \\
$\mathrm{CV}(\%)$ & 11,42 & \\
\hline
\end{tabular}

*As médias não seguidas pela mesma letra, na coluna, diferem entre si em nível de 5\% de probabilidade de erro pelo teste de Tukey.

\section{AGRADECIMENTOS}

Ao Programa Institucional de Bolsas de Iniciação Científica do Conselho de Desenvolvimento Científico e Tecnológico (PIBIC/CNPq/UFSM), pela concessão de bolsa de iniciação científica (BIC) ao estudante do Curso de Agronomia da UFSM Roberto Trentin. À estudante do Curso de Agronomia da UFSM Eloiza Lasta, pela ajuda na condução do experimento e na coleta dos dados.

\section{REFERÊNCIAS}

ANDRIOLO, J.L. Fisiologia das culturas protegidas. Santa Maria: UFSM, 1999. 142p.

AZKUE, M.P. de; PUCHE, M. La temperatura como herramienta de predicción agroclimatológica aplicada a la producción de frutales. Revista Digital del Centro Nacional de Investigaciones Agropecuarias de Venezuela. n.3, septiembre - diciembre, 2003. Capturado em 18 abr. de 2006. Online. Disponível em: www.ceniap.gov.ve/ceniaphoy/articulos/ n3/texto/mazkue.htm

BAKER, J.T.; REDDY, V.R. Temperature effects on phenological development and yield of muskmelon. Annals of Botany, v.87, n.5, p.605-613, 2001.

BAKER J.T. et al. Effects of temperature on leaf appearance in spring and winter wheat cultivars. Agronomy Journal, v.78, n.4, p.605-613, 1986.

BAUMANN, L.S. Cosmeceutical critique: calendula. (Dermatologic Therapy). Skin \& Allergy News,. v.34, n.10, p.17, 2003.

BERTI, M.D. et al. Influencia de la fecha de siembra y de la procedência de la semilla em el rendimiento de capítulos de Calendula officinalis L. durante dos temporadas em Chillán. Agricultura Técnica, v.63, n.1, p.3-9, 2003.

CROMACK, H.T.H.; SMITH, J.M. Calendula officinalis production potential and crop agronomy in southern England. Industrial Crops and Products, v.7, n.2, p.223-229, 1998.

DELLA LOGGIA, R. et al. The role of triterpenoids in the topical anti-inflammatory activity of Calendula officinalis L. Flower. Planta Medica, v. 60, p.516-520, 1994.
DERKSEN, J.T.P. et al. Paints and coatings from renewable resources. Industrial Crops and Products, v.3, n.4, p.225$236,1995$.

ELLIS, R.P.; RUSSELL, G. Plant development and grain yield in spring and winter barley. Journal of Agricultural Science, v.102, p.85-95, 1984.

FRANK, A.B.; BAUER, A. Phyllochron differences in wheat, barley and forage grasses. Crop Science, v.35, n.1, p.19-23, 1995.

HERMES, C.C. et al. Emissão de folhas de alface em função da soma térmica. Revista Brasileira de Agrometeorologia, v.9, n.2, p.269-275, 2001.

JEFFERIES, R.A. et al. Thermal time as a non-destructive method of estimating tuber initiation in potatoes. Journal of Agricultural Science, v.108, n.1, p.249-252, 1987.

KALVATCHEV, Z. et al. Anti-HIV activity of extracts from Calendula officinalis flowers. Biomed \& Pharmacother, v.51, n.4, p.176-180, 1997.

KIRBY, E.J.M. et al. Effect of sowing date and variety on main shoot leaf emergence and number of leaves of barley and wheat. Agronomie, v.5, n.2, p.117-126, 1985.

KLEPPER, B. et al. Quantitative characterization of vegetative development in small cereal grains. Agronomy Journal, v.74, n.5, p.789-792, 1982 .

MUCHOW, R.C; CARBERRY, P.S. Phenology and leaf - area and development in a tropical grain sorghum. Field Crops Research, v.23, n.3, p.221-237, 1990.

MUUSE, B.G. et al. Composition and physical properties of oils from new oilseed crops. Industrial Crops and Products, v.1, p.57-65, 1992.

PAULA, F.L.M. et al. Filocrono da planta de batata cultivar Asterix em diferentes épocas de plantio. Revista Brasileira de Agrometeorologia, v.13, n.3, p.367-374, 2005.

SLAFER, G.A. et al. Rate of leaf appearance and final number of leaves in wheat: effects of duration an rate of change of photoperiod. Annals of Botany, v.74, n.5, p.427-436, 1994.

STRECK, N.A. A generalized nonlinear air temperature response function for node appearance rate in muskmelon (Cucumis melo L.). Revista Brasileira de Agrometeorologia, v.10, n.1, p.105-111, 2002.

STRECK, N.A. et al. Incorporating a chronology response into the prediction of leaf appearance rate in winter wheat. Annals of Botany, v.92, n.2, p.181-190, 2003a.

STRECK, N.A. et al. Improving predictions of developmental stages in winter wheat: A modified Wang and Engel model. Agricultural and Forest Meteorology, v.115, n.3, p.139$150,2003 \mathrm{~b}$.

XUE, Q. et al. Predicting leaf appearance in field-grown winter wheat: e valuating linear and non-linear models. Ecological Modelling, v.175, n.3, p. 261-270, 2004. 